\title{
Unconscious Design and the Natural Qualities of Bamboo Furniture
}

\author{
Wenying Dong ${ }^{1, \star}$, Jiamin Huang ${ }^{1}$ \\ ${ }^{1}$ Forestry College, Sichuan Agricultural University, Chengdu 611134, China
}

\begin{abstract}
Combined with the features of bamboo, to discuss how to release the qualities of bamboo using the unconscious design skillfully, and show "humanism" of bamboo furniture to achieve harmony between man, machine and environment.
\end{abstract}

\section{Introduction}

People's thought and spirit is becoming numb in front of the more and more cruel competition for survival. In people's subconscious, they desire to live in a pure and simple environment, and with the arrival of the era of perceptual consumption, the value of commodities added emotional factors has became a new benchmark to determine the quality. Being aware of such a situation, modern designers however emphasize the "differentiation" of products by diversifying the shape or function, and they stiffly add some elements such as "natural" and "simple" to the innovative design of products. They ignore the users irrational preference and even conflict with new products, so these products increase the "adaptive burden" for users. How to make people use bamboo furniture naturally, and highlight the "simplicity" of the bamboo's natural qualities at the same time? How to unify the interior modern pattern with bamboo furniture? Fukazawa Naoto's conception of Unconscious design make them perfectly dissolve with each other in the solution of the innovative design. ( Figure 1)

\section{Innovation points}

The noble and pure character of bamboo parallels to the simplicity in people's subconscious. Based on the concept of the unconscious design, process bamboo as a raw material to show the fresh, elegant character. It will create more freedom and variable factors for users, so this ideology which is similar with the theory of Hara Kenya

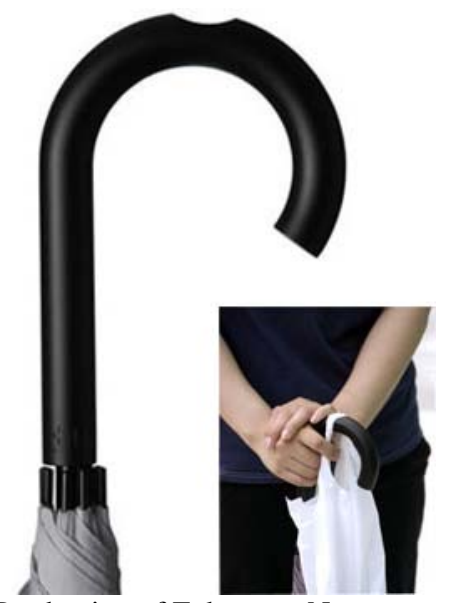

Figure 1. Production of Fukazawa Naoto---a umbrella with groove that meets implicit demand of users who pointed that "white is empty" in Zen idea, but rather can accept the ideas of all people with wide inclusiveness. This kind of bamboo furniture that achieves the unconscious behaviors of users by conscious design of designers meets the implicit demand of users, and wakes up the collective memory of masses from inside the material essence to outside the implicit emotion, so it effectively eliminates the gap between people and products. Throughout the product market of bamboo furniture in China, based more on bamboo furniture with Chinese style or integrated composite bamboo furniture, and the methods showing the natural and simple qualities of bamboo furniture are so far-fetched. In this circumstances, the design of bamboo furniture combined with the unconscious design will become a new way to thoroughly release the natural qualities of bamboo.

\section{The innovative application of unconscious design to bamboo furniture}

By means of a number of cases, unconscious design can discover the development trend of things and further sum up the corresponding rules, and finally solve the problems of product defects that users are difficult to clearly put forward and even perceive. Such products as take people's requirements into account can only succeed in the long-term competitive merchandise market. Therefore, it not only restores the original characteristics of bamboo furniture, but also doesn't deviate from the stream of consciousness of majority groups, to carry out innovative designs of bamboo furniture in way of combining the features of the bamboo itself based on the leading principle idea of unconscious design. 


\subsection{Adhere to the principle of unconscious design---Pay attention to consciousness, and zoom in details}

Design inspiration comes from the attention to consciousness and the observation to life details. It collects mass unconscious behaviors of people in the details, from which it extracts shared elements, enlarges details and injects them into ordinary products anew, then achieve so-called "just OK in this" products that agree with unconscious need of users. To a good product, detail may be a chamfer, a line, kind of applying of material or a change of usage mode. These details are not only the key for products to withstand scrutiny, but also the base of design innovation. They are significant portions of achieving extrinsic value of product ${ }^{[1]}$. In modern society, most users subconsciously prefer to simple material, and bamboo is the concretion of collective unconsciousness concerning the details. For this reason, in the process of designing furniture, it is supposed to recover the natural functions of bamboo as much as we can, including texture, color, feeling and so on, and focus on displaying true nature of bamboo naturally. This is the best consideration for collective unconscious preference.

\subsection{Show the natural qualities of bamboo combined with the unconscious design}

The obvious characteristics of unconscious design lies in its rational grasp and organic fusion of "minimalism", "humanism" as well as the man-machine harmonious interactive environment. Combined with the idea of the unconscious design, it highlights the true qualities of bamboo such as plain, aloof, quietly elegant, and peaceful completely, basing on bamboo furniture design.

\subsection{1 the "Minimalism" highlights the rustic material of bamboo}

Japanese aesthetic art pursues "Elegant" spirit. The "Elegant" means neither giving up comfort or aesthetic pleasure nor showing off too many details or cumbersome adornment. It focus on the smallest, and also lays great stress on the appreciation of material and the faith in nature ${ }^{[[2]]}$.

Bamboo is enjoyed by people with its fresh fragrance and the smooth sense of touch. Considering bamboo as the raw material which is frugal but not simple to make all users understand the connotation of products no matter who they are is an effectively way to activate the cognitive mode of mass unconsciousness. Therefore, do follow the simple rules to arrange the bamboo into a whole orderly, and do stay the natural attributes of bamboo such as cool, smooth, fragrance, when faced with the design of bamboo. What's more, in order to maintain the original style of furniture, designers should emphasize the harmonious proportion on the minimizing of form, and the maximizing of the function to simplify the unnecessary decoration so that it can make the bamboo furniture go back to nature. (Figure 2)

\subsubsection{The implicit demand reflects the strong integrity of bamboo}

In the field of product design, the user's demand is not clear, having characteristic as ambiguity, implicit, and dynamic $^{[3]}$. However the design is neither to find

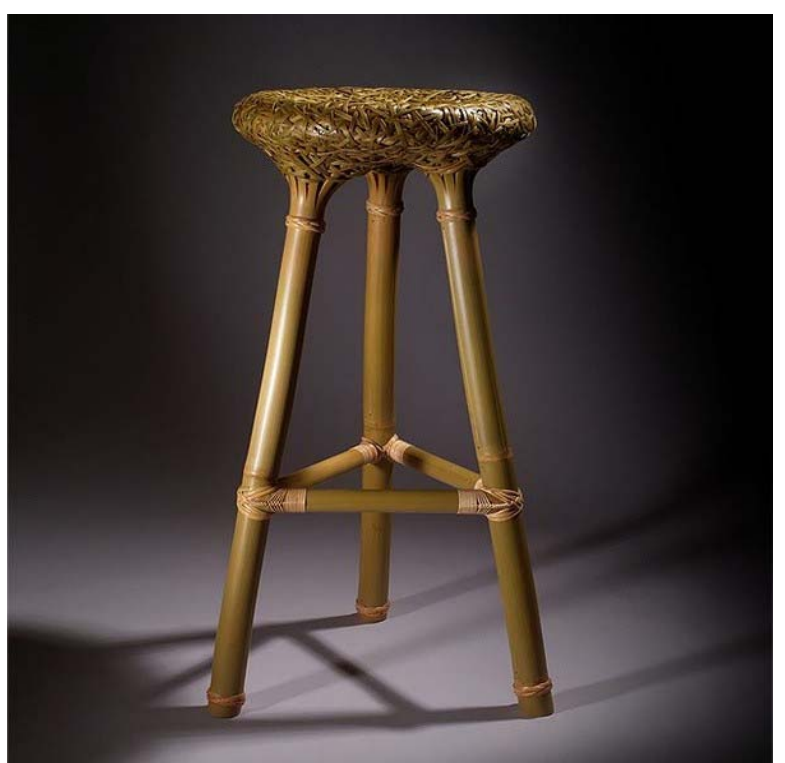

Figure 2.A stool----highlights the rustic material of bamboo

demand simply nor create demand blindly, but to present the things that already exist in people's subconscious with a beautiful form, giving them chance to meet each other ${ }^{[4]}$. In the design of bamboo furniture, needing design perfect unconscious work according users imperceptible behavioral results, and rely on users' unconscious work "without thinking" to finish bamboo furniture's function. While without thinking's intensity is not clear, for essential furniture's function, needing to bear the action of the unknown, and require the material hardness, toughness, and other basic properties can provide enough support. The reason why bamboo has become the furniture industry's environmental protection materials replacing wood, whether in the form of linear circular bamboo, or plate form, its specific parts of the anti-tensile, anti-compression, anti-flex strength and corrosion resistance and wear resistance are higher than the wood. The characteristics of bamboo not only ensure the quality

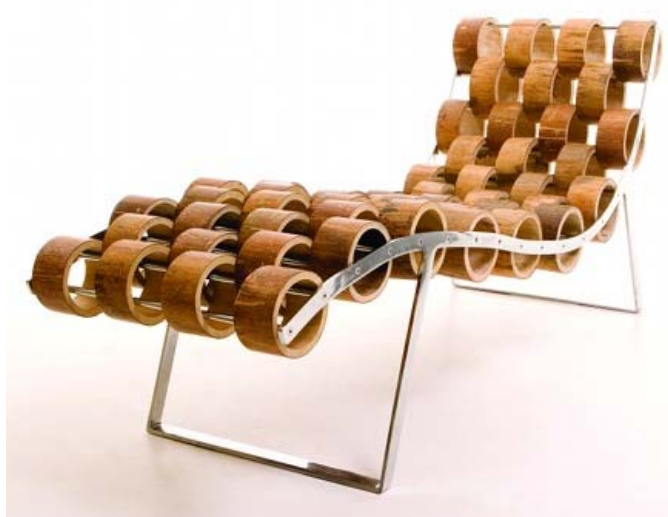

Figure 3.A massage chair---- reflects the excellent performances of bamboo 
of the user's implicit needs meet also reflects its hard and strong integrity. (Figure 3)

\subsubsection{The emotional resonance represents the elegant poetry of bamboo}

The need of user's unconscious is the most natural expression of human emotion ${ }^{[[5]]}$. Seizing the real emotion will make bamboo furniture attach to the emotion of independence, when the brain contain similar conscious experience of individual users with any one of the five senses attacked, there will be produced emotions intertwined which are out of control, and fragments conscious of life experience are associated orderly and awaken by the unconscious bamboo furniture, which will make users can experience the subconscious needs or sweet memories which can get the respond in product design happily, and have a strong resonance.

In the Chinese traditional system of thinking, bamboo with its nobility and tough exist in the culture of poetry, with its simplicity and nature exist in a quiet countryside, with its fresh and comfort exist in childhood memories. These "existence" are what the people yearning for in the modern society. Let the bamboo furniture of unconscious design concept stimulate a point of the collective consciousness, so that the users can generate stress behaviors in the aspects of bamboo furniture's function and emotions. During the decode procedure of bamboo furniture, users integrate into their own understanding and emotion, and in the meantime their nature and simplicity emotions also will integrate into the bamboo furniture.

\subsubsection{The harmony of man-machine-environment shows the quiet and peaceful character of bamboo}

Products are not only measured by the standards of single dimension of artistic facade, practicability, easily use, humanization, friendly environment, technical innovation, but a complex of all. And they keep a good relationship with both users and environment, which appear when needed, disappear when there's no need. Products have no signs of deliberately "design", but bring natural experience beyond visual interest ${ }^{[6]}$, this is called "the hub out of ordinary". Therefore, the most striking design is not to please the masses through the creative ideas, but to consider the "people - machine - environment" as a whole, to achieve harmony.

Since ancient time, people have been giving a high praise to bamboo for its elegant nature and integrity of inoffensive. During reducing natural bamboo furniture design process, follow the unconscious design principle of harmonious balance, which is consistent with Japanese architect Kengo Kuma's ideas, which is letting bamboo furniture can disappear visually in the environment, but its function is inevitable in the environment of transparency. This transparency is not merely a continuous of vision, but a combination of modern hightech and regional natural material, which is a attempt of combination of traditional, regional, apex and global.

\section{The prospect of bamboo furniture guided by the unconscious design system of China}

The concepts such as the "Unconscious design" of Fukazawa Naoto's, the "Unconscious design" of Kengo Kuma, the "Anti-planning" of Kongjian $\mathrm{Yu}$ who is the domestic landscape planners, and the "Inverse planning" that exist already, are the appropriate applications of the "Reverse thinking" to reduce the design burden for users, and make users experience the convenience and pleasure of design without consciousness. The thought of "Inaction" of Chinese is similar with the "Zen culture" of Japan, and both of them have reached a certain height on ideology. Add these concepts into the design of bamboo furniture and the connotation of the products will be more abundant. Meanwhile, these concepts back to the starting point of design emphasize that the design is not to show off the skills. However, it will go to the opposite direction if regard the "Unconscious design"and "Anti-planning" as the design without consciousness and planning ${ }^{[7]}$.

On the design road of China, it is necessary to learn from foreign outstanding cases and concepts with which to develop a completely concept system of design based upon china's conditions. Japan had rapidly recovered its economy after World War II followed by the development industrial design. Japanese designers gradually form a new innovative path rather than the imitation before, so the style of Japan's design become one of the leading direction of global design now. As one of the progressive concept of Japan, the unconscious design goes with the tide of social development. The design of bamboo furniture guided by the ideas of the unconscious design is a resulting present of analyzing all the aspects of demand, so it naturally shows unconscious behaviors of users, and also effectively reduces user's subconscious conflict with emerging products. This bamboo furniture keeps a long-term and stable relationship with people, and promote the construction of harmonious society. Lastly, the bamboo furniture design of China will gradually come out a new research road relying on the concept of the unconscious design.

\section{Conclusion}

Combining with the unconscious design, the Bamboo furniture has the following characteristics: First of all, in terms of industrial design or literary trend of thought, bamboo in China all seem to give the fresh and natural, unpretentious impression to humans, and can produce strong regional flavor with Chinese cultural elements, named "Regional". Secondly, the unconscious design concept used in bamboo furniture design, abandoning complicated, reducing the difficulty of information received, is bringing "fresh wind" to the fast-paced world, which conform the global flavors, named "Global". Thirdly, mixing the pivotal function of bamboo furniture with user's potential behaviors, touching users' deepest needs, which aim to establish the positive interaction between bamboo furniture and users, reflecting on the humane care of bamboo furniture, named "Humanism". 
The bamboo furniture integrating these three factors organically is the perfect combination performance of emotional and rational design, which not only inherit and carry forward the traditional bamboo culture of our nation, but also reflects the modern international tastes of bamboo furniture. This "Harmonious but Different" design combination improves the market competitiveness of bamboo furniture, and also conforms to the trend of globalization. In promoting environmental topics, let the actual unconscious design play a more actual effect and guide the users' green behavior, which could quietly develop their good habits and create a green ecological environment. (Figure 4).

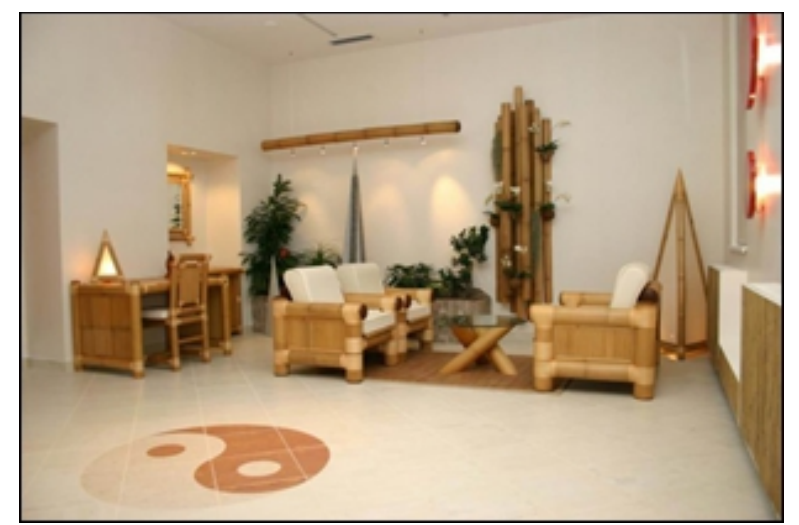

Figure 4.The harmony of man-machine environment

\section{Acknowledgements}

This work was financially supported by the research program of Allied Social Science Association of Sichuan Agricultural University (2013YB01).

\section{References}

[1] X.J. He, W. F. Pan, The simple beauty and research of the unconscious design [J]. Yihai (2014)11:84-85

[2] Y. Z. Li, Foreign design art classic reader (below) [M]. Beijing: Tsinghua University Press(2006):15 20

[3] P. Duan, Analysis of product design based on the subconscious thinking [J]. Decoration(2010)02:7677

[4] Y. N. Huang, Back to the origin of product design -Analysis of Fukazawa Naoto's idea and design practice [J]. Decoration(2008)11:112-114

[5] J. Zhang, The application research of the unconscious design in interactive design [J]. Education Guide(2012)07:142-143+201

[6] Y. Gao, To grasp the desire of freedom -- Talk on the unconscious design from the perspective of experience [J]. Art and design(theory)(2011)02:1415

[7] M. R. Xie, H. L.Duan, The concept formation of unconscious design and the development research[J]. Jian'nan literature(2012)06:212. 